\title{
Kane Method Based Dynamics Modeling and Control Study for Space Manipulator Capturing a Space Target
}

\author{
Yanhua Han \\ College of Astronautics, Nanjing University of Aeronautics and Astronautics, Nanjing 210016, China \\ Correspondence should be addressed to Yanhua Han; hanyanhua@nuaa.edu.cn
}

Received 29 January 2016; Revised 3 June 2016; Accepted 15 June 2016

Academic Editor: Christopher J. Damaren

Copyright ( 2016 Yanhua Han. This is an open access article distributed under the Creative Commons Attribution License, which permits unrestricted use, distribution, and reproduction in any medium, provided the original work is properly cited.

\begin{abstract}
Dynamics modeling and control problem of a two-link manipulator mounted on a spacecraft (so-called carrier) freely flying around a space target on earth's circular orbit is studied in the paper. The influence of the carrier's relative movement on its manipulator is considered in dynamics modeling; nevertheless, that of the manipulator on its carrier is neglected with the assumption that the mass and inertia moment of the manipulator is far less than that of the carrier. Meanwhile, we suppose that the attitude control system of the carrier guarantees its side on which the manipulator is mounted points accurately always the space target during approaching operation. The ideal constraint forces can be out of consideration in dynamics modeling as Kane method is used. The path functions of the manipulator's end-effector approaching the space target as well as the manipulator's joints control torque functions are programmed to meet the soft touch requirement that the end-effector's relative velocity to the space target is zero at touch moment. Numerical simulation validation is conducted finally.
\end{abstract}

\section{Introduction}

Tethered space robot and space manipulator are two means for on-orbit operation such as satellite maintenance, refueling, retrieval, or space debris removal. The main advantage of the former over the latter is its long operation distance (from tens of meters to dozens of kilometers), while the disadvantage of the former is obvious such as inherent instability in retrieval procedure and huge difficulties in control design for the procedure [1]. Compared to tethered space robot, space manipulator can work efficiently within the scope of tens of meters or smaller [2,3], such as SRMS (Shuttle Remote Manipulator System) [2].

Generally, the system dynamics of space manipulator is more complex than that on the ground $[4,5]$. In the field of space manipulator dynamics, [6] presents the singularityfree dynamic equations of the dynamically equivalent manipulator (DEM) of spacecraft-manipulator systems, which is computationally efficient and meanwhile possesses the same dynamic properties as the corresponding free-floating spacecraft-manipulator system. Reference [7] outlines a procedure for modeling three-dimensional flexible multibody manipulators during maneuvering and payload capture. The manipulators are capable of performing large rigid-body translations and rotations, as well as experiencing deformation as a result of their inherent flexibility. This motion is highly nonlinear, so a nonlinear corotational finite element method is used. Reference [8] presents a study to understand the true dynamics of the tested space manipulator aiming at the problem encountered in ground physical simulation that the air-bearing support system changes the dynamics characteristics of the manipulator such as the natural frequencies, stiffness, and damping. References [9-11] study the configuration, dynamics, and control problem of a tendonactuated lightweight space manipulator.

Trajectory optimization of manipulator with redundant degree of freedom for capturing space target is researched in $[12,13]$ with the influence of manipulator's movement on its carrier taken into account. An improved manipulator trajectory optimization method for such system with nonconserved momentum and angular momentum is presented in [14]. The method allows minimization of a quadratic norm connected with the power use of motors in manipulator 
joints. The method differs from its previous version in including the possibility of constraint final velocity of the endeffector and by considering period of the capture maneuver as a parameter that is optimized.

The closed loop control law is designed for manipulator when approaching and capturing space target in $[15,16]$. In [15], the flexibility of manipulator's joints is considered, and then the notion of artificial potential is introduced to design the control law so as to guarantee the stability of the closed loop, while linear quadratic control theory is adopted in [16]. In [17], contact mechanics between the end-effector of manipulator and space target is taken into account, and the dynamics for the whole procedure is modeled; then the control law is designed. Reference [18] has discussed the control strategies for the autonomous target capture of a freeflying space robot. The highlight is made on the reactionless manipulation and the generalized Jacobian matrix. The reactionless manipulation is obtained from the reaction nullspace (RNS) of the coupling inertia matrix that describes the dynamic interaction between the manipulator and the base attitude motions. The obtained manipulator motion yields zero attitude disturbance on the base satellite. Reference [19] presents a method for control of micro-macro manipulators. The method splits the control problem into two parts. The first part is to devise a controller which moves the macro manipulator close to the desired path, and the second part is to design a controller to enforce the tracking error of the end-effector to zero. The main advantage of the controller is that it does not try to prevent fast motion of macro manipulator to avoid excitation of higher modes. In [20], fuzzy logic is applied to control a novel two-link robotic arm. The control system has three levels. A conventional controller consisting of the feedback linearization technique combined with proportional-derivative control is used in the bottom level of the hierarchical system to control the servomotors of the robot. A second layer consists of a servo-expert that preprocesses the high-resolution information coming from joint encoders and extracts the status of the system. A third, intelligent layer is added at the top of the hierarchy to complete the control structure. The main purpose of the top level is to tune the parameters of the conventional controller to improve the response of the system.

For tethered space robot dynamics and control problem, the involved orbit dynamics is taken into account adequately [21-30]. In [21-25], the main satellite or shuttle (from which the tethered space robot is released out) is on circular orbit around the earth, while in [26-30], the influence of the eccentricity of the main satellite's ellipse orbit on the dynamics of the tethered system is studied. Compared to the tethered space robot, almost all the dynamics modeling for space manipulator presented in documented literatures to date is conducted with respect to inertial reference system; that is to say, the influence of the relative orbit dynamics between the carrier and the target on the system dynamics modeling is not taken into account. The paper attempts to derive the dynamics model of two-link manipulator when its end-effector approaches target based on Kane method with respect to the carrier body-fixed noninertial coordinate system; that is to say, the relative orbit dynamics of the carrier to the space target is considered in system dynamics modeling. Then the end-effector's path function and the manipulator's corresponding joints control torque function are programmed to meet the requirement of soft touch, which means that the relative velocity of the end-effector to the target is zero at touch moment. Finally, numerical simulation will be conducted to validate the work done in the paper.

\section{System Dynamics Modeling}

2.1. Preliminary Work. A space target is supposed to fly on a circular orbit around the earth. An operation spacecraft (called carrier uniformly afterwards in the paper) flies freely around the target governed by the well-known homogenous $\mathrm{C}-\mathrm{W}$ equations [31], as shown in Figure 1. In the figure, the origin of coordinate system $o x_{A} y_{A}$ denotes the space target. Axis $o x_{A}$ points the direction of the velocity of the space target relative to the earth's center, and axis $o y_{A}$ lies on the extended line from the earth's center to the space target with the positive direction deviating from the earth. The ellipse denotes the relative trajectory of the carrier to the target and is supposed to be coplanar with the space target's circular orbit around the earth. The direction of the carrier flying around the target in Figure 1 should be anticlockwise according to C$\mathrm{W}$ equations. Two links of the manipulator are identified as I and II. Their mass, moment of inertia with respect to their centers of mass, length, and angle of rotation are $m_{i}, J_{i}, l_{i}, \theta_{i}$ ( $i=1,2$ corresponding to $\mathrm{I}, \mathrm{II}$ ), respectively, and both of the two links are assumed to be homogenous in mass. Two motors are mounted on the joints identified as I and II, and their driving torques are $M_{1}$ and $M_{2}$, respectively. The distance between joint I and the center of mass of the carrier is $d$. The assumption that the mass and inertia moment of the carrier is far bigger than that of the manipulator is adopted here, and then the influence of the manipulator's movement to the carrier can be ignored. We also suppose that the attitude control system of the carrier guarantees its side on which the manipulator is mounted points accurately always the space target during approaching operation.

Some coordinate systems and the corresponding unit orthogonal basis are defined as follows:

(1) Earth-centered inertial coordinate system $o x_{I} y_{I}$ : the origin is at the center of the earth. Axis $o x_{I}$ points a fixed direction in inertia space. Axis $o y_{\mathrm{I}}$ is got by rotating axis $o x_{\mathrm{I}}$ with $90^{\circ}$ anticlockwise. $\vec{i}_{1}$ and $\vec{i}_{2}$ are unit vectors lying on axes $o x_{\mathrm{I}}$ and $o y_{\mathrm{I}}$, respectively, and their directions are the same as the positive directions of the corresponding axes, respectively.

(2) Space target fixed coordinate system $o x_{A} y_{A}$ : being defined above, it will not be repeated here. $\vec{a}_{1}$ and $\vec{a}_{2}$ are unit vectors lying on axes $o x_{A}$ and $o y_{A}$, respectively, and their directions are the same as the positive directions of the corresponding axes, respectively.

(3) Carrier body-fixed coordinate system $o x_{B} y_{B}$ : the origin is at the center of mass of the carrier. Axis $o x_{B}$ lies on the extended line from the space target to the carrier with the positive direction deviating from the space target. Axis $o y_{B}$ is got by rotating axis 


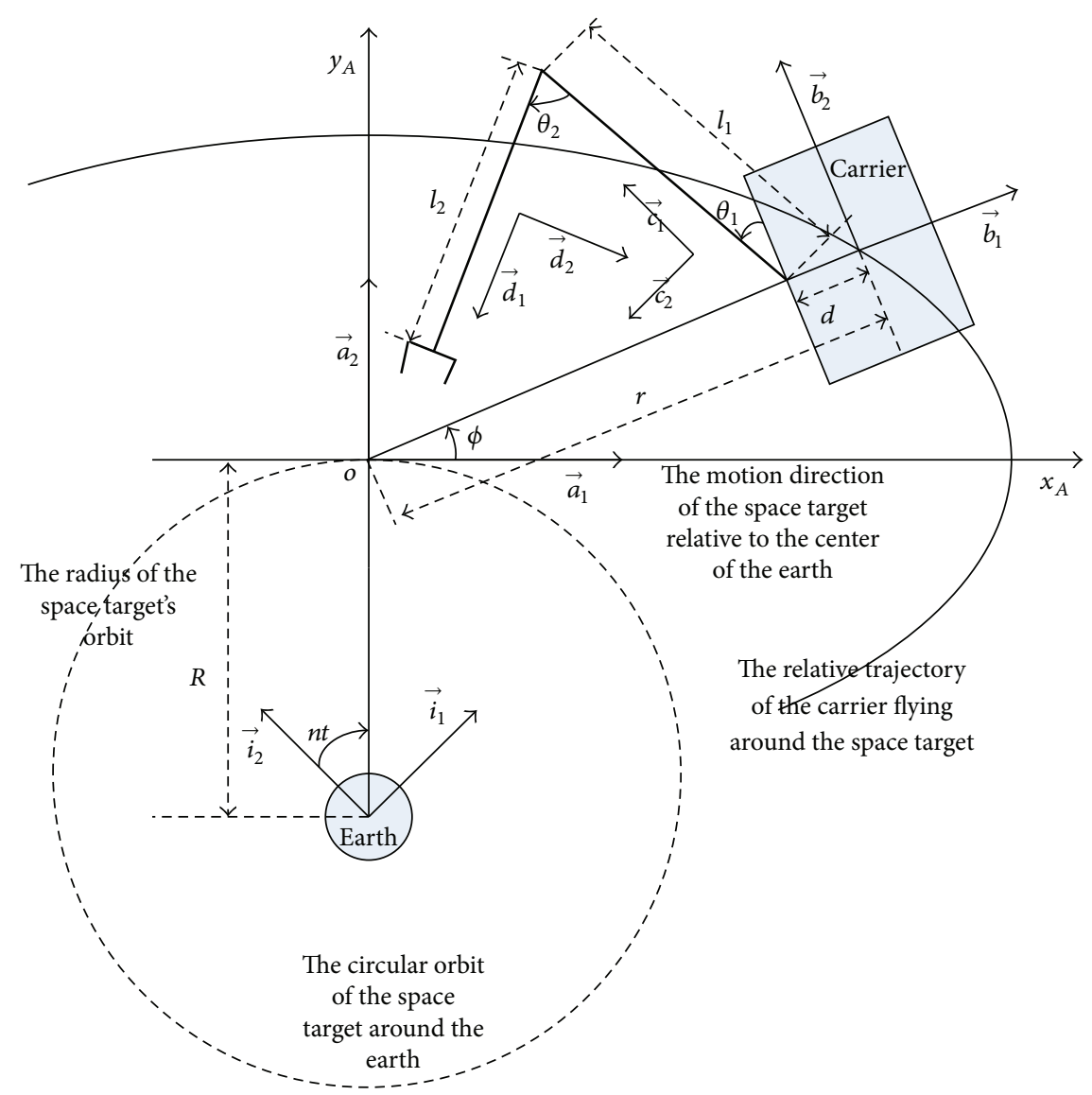

FIGURE 1: Illustration of space manipulator capturing an on-orbit target around the earth.

$o x_{B}$ with $90^{\circ}$ anticlockwise. $\vec{b}_{1}$ and $\vec{b}_{2}$ are unit vectors lying on axes $o x_{B}$ and $o y_{B}$, respectively, and their directions are the same as the positive directions of the corresponding axes, respectively.

(4) Link I fixed coordinate system $o x_{C} y_{C}$ : the origin is at the center of mass of the link I. Axis $o x_{C}$ is aligned with the longitudinal direction of the link with the positive direction deviating from joint I. Axis $o y_{C}$ is got by rotating axis $o x_{C}$ with $90^{\circ}$ anticlockwise. $\vec{c}_{1}$ and $\vec{c}_{2}$ are unit vectors lying on axes ox $x_{C}$ and $o y_{C}$, respectively, and their directions are the same as the positive directions of the corresponding axes, respectively.

(5) Link II fixed coordinate system $o x_{D} y_{D}$ : the origin is at the center of mass of the link II. Axis $o x_{D}$ is aligned with the longitudinal direction of the link with the positive direction deviating from joint II. Axis $o y_{D}$ is got by rotating axis $o x_{D}$ with $90^{\circ}$ anticlockwise. $\vec{d}_{1}$ and $\vec{d}_{2}$ are unit vectors lying on axes $o x_{D}$ and $o y_{D}$, respectively, and their directions are the same as the positive directions of the corresponding axes, respectively.

In accordance with the above definition, the vector groups $\mathbf{I} \triangleq\left(\vec{i}_{1}, \vec{i}_{2}\right), \mathbf{A} \triangleq\left(\vec{a}_{1}, \vec{a}_{2}\right), \mathbf{B} \triangleq\left(\vec{b}_{1}, \vec{b}_{2}\right), \mathbf{C} \triangleq\left(\vec{c}_{1}, \vec{c}_{2}\right), \mathbf{D} \triangleq$ $\left(\vec{d}_{1}, \vec{d}_{2}\right)$ are all unit orthogonal basis vector groups with the transformation relations among them as follows:

$$
\begin{aligned}
& \mathbf{A}=\mathbf{I T}_{\mathrm{IA}}, \\
& \mathbf{B}=\mathbf{A T}_{\mathrm{AB}}, \\
& \mathbf{C}=\mathbf{B T}_{\mathbf{B C}}, \\
& \mathbf{D}=\mathbf{C T}_{\mathbf{C D}}, \\
& \mathbf{B}=\mathbf{I T}_{\mathrm{IA}} \cdot \mathbf{T}_{\mathbf{A B}}=\mathbf{I T}_{\mathrm{IB}},
\end{aligned}
$$

where the transformation matrixes are as follows:

$$
\begin{aligned}
\mathbf{T}_{\mathbf{I A}} & =\left(\begin{array}{cc}
\cos n t & \sin n t \\
-\sin n t & \cos n t
\end{array}\right), \\
\mathbf{T}_{\mathbf{A B}} & =\left(\begin{array}{cc}
\cos \phi & -\sin \phi \\
\sin \phi & \cos \phi
\end{array}\right), \\
\mathbf{T}_{\mathbf{B C}} & =\left(\begin{array}{cc}
-\sin \theta_{1} & -\cos \theta_{1} \\
\cos \theta_{1} & -\sin \theta_{1}
\end{array}\right), \\
\mathbf{T}_{\mathbf{C D}} & =\left(\begin{array}{cc}
-\cos \theta_{2} & -\sin \theta_{2} \\
\sin \theta_{2} & -\cos \theta_{2}
\end{array}\right),
\end{aligned}
$$




$$
\mathbf{T}_{\mathbf{I B}}=\mathbf{T}_{\mathbf{I A}} \cdot \mathbf{T}_{\mathbf{A B}}=\left[\begin{array}{cc}
\cos (n t-\phi) & \sin (n t-\phi) \\
-\sin (n t-\phi) & \cos (n t-\phi)
\end{array}\right] .
$$
ingly:

The following equations can be easily obtained accord-

$$
\mathbf{T}_{\mathbf{I B}}{ }^{T} \cdot \dot{\mathbf{T}}_{\mathbf{I B}}=\left(\begin{array}{cc}
0 & n-\dot{\phi} \\
\dot{\phi}-n & 0
\end{array}\right) .
$$

What should be pointed out is that a vector keeps its value when being moved in parallel. So unit orthogonal basis of vector groups $\mathbf{C}$ and $\mathbf{D}$ can be moved in parallel departing from their corresponding coordinate systems $o x_{C} y_{C}$ and $o x_{D} y_{D}$, as shown in Figure 1.

In Figure 1, coordinate system $o x_{A} y_{A}$ is fixed upon the space target and moves around the earth at a constant angular velocity $n=\sqrt{\mu / R^{3}}$, where $\mu=G M_{e}$, and $G, M_{e}, R$ are gravitational constant, the mass of the earth, and the radius of the space target's circular orbit, respectively. We know that with a group of specific initial conditions the relative trajectory of the carrier to the space target is an ellipse with eccentricity ratio of $\sqrt{3} / 2$, and the relative motion equations are as follows under the coordinate system $o x_{A} y_{A}[31]$ :

$$
\begin{aligned}
& \ddot{x}=-2 n \dot{y}, \\
& \ddot{y}=3 n^{2} y+2 n \dot{x}, \\
& \dot{x}=-2 n y_{0} \cos n t-n x_{0} \sin n t, \\
& \dot{y}=\frac{n}{2} x_{0} \cos n t-n y_{0} \sin n t, \\
& x=x_{0} \cos n t-2 y_{0} \sin n t, \\
& y=y_{0} \cos n t+\frac{x_{0}}{2} \sin n t,
\end{aligned}
$$

where $x_{0}, y_{0}$ denote the position coordinates of the carrier relative to the target at initial moment $(t=0)$.

The following equations can be obtained easily:

$$
\begin{aligned}
r & =\sqrt{x^{2}+y^{2}}, \\
\phi & =\arctan \frac{y}{x} \text { (appropriate for first quadrant), } \\
\dot{r} & =\dot{x} \cos \phi+\dot{y} \sin \phi, \\
\dot{\phi} & =\frac{-\dot{x} \sin \phi+\dot{y} \cos \phi}{r}, \\
\ddot{r} & =r \dot{\phi}^{2}+\ddot{x} \cos \phi+\ddot{y} \sin \phi, \\
\ddot{\phi} & =\frac{-2 \dot{r} \dot{\phi}-\ddot{x} \sin \phi+\ddot{y} \cos \phi}{r},
\end{aligned}
$$

where $r$ is the distance between the center of the carrier and the space target and $\phi$ is the phase angle of the carrier flying around the target.

We know the origin of coordinate system $o x_{A} y_{A}$ moves around the earth at angular velocity $n$; meanwhile, the coordinate system rotates about its own origin at the same angular velocity. So the coordinate system is noninertial. Nevertheless, its translational movement around the earth as well as the gravities exerted on the objects under the coordinate system (which are the manipulator and its carrier) can be out of consideration simultaneously, since the translational movement of the coordinate system originates from the gravity. So the equivalent centrifugal forces exerted on the objects under the coordinate system resulted from the coordinate system's circle movement counteract exactly the corresponding gravities of them. However the rotation of coordinate system $o x_{A} y_{A}$ about its own origin should be considered in dynamics modeling. Coordinate system $o x_{B} y_{B}$ moves around the space target (the trajectory is an ellipse) and meanwhile rotates about its own origin at angular velocity $\dot{\phi}$. According to the analysis above, the linear acceleration of coordinate system $o x_{B} y_{B}$ relative to $o x_{A} y_{A}$ can be regarded as that relative to inertial reference system; meanwhile, the angular velocity of coordinate system $o x_{B} y_{B}$ relative to coordinate system $o x_{A} y_{A}$ (it is $\dot{\phi}$ ) minus that of $o x_{A} y_{A}$ relative to inertial reference system (it is $n$ ) is equal to that of $o x_{B} y_{B}$ relative to inertial reference system. Obviously it is $\dot{\phi}-n$. So the effects of the linear acceleration of coordinate system $o x_{B} y_{B}$ relative to $o x_{A} y_{A}$ and its rotation angular velocity relative to inertial reference system should be considered in system dynamics modeling.

2.2. Analysis of Kinematics and Mechanics. The position vector of the origin of coordinate system $o x_{B} y_{B}$ relative to the space target is

$$
\vec{r}=\mathbf{B}\left(\begin{array}{l}
r \\
0
\end{array}\right) .
$$

The time derivative of $(8)$ is

$$
\vec{v} \triangleq \frac{\mathrm{d} \vec{r}}{\mathrm{~d} t}=\frac{\mathrm{d} \mathbf{B}}{\mathrm{d} t}\left(\begin{array}{l}
r \\
0
\end{array}\right)+\mathbf{B}\left(\begin{array}{l}
\dot{r} \\
0
\end{array}\right) .
$$

In the same way, the time derivative of (9) is

$$
\vec{a} \triangleq \frac{\mathrm{d} \vec{v}}{\mathrm{~d} t}=\frac{\mathrm{d}^{2} \mathbf{B}}{\mathrm{d} t^{2}}\left(\begin{array}{l}
r \\
0
\end{array}\right)+2 \frac{\mathrm{d} \mathbf{B}}{\mathrm{d} t}\left(\begin{array}{l}
\dot{r} \\
0
\end{array}\right)+\mathbf{B}\left(\begin{array}{l}
\ddot{r} \\
0
\end{array}\right),
$$

where $\vec{v}$ and $\vec{a}$ are the velocity and acceleration of the origin of coordinate system $o x_{B} y_{B}$ relative to the space target, respectively. According to (2), we obtain

$$
\begin{aligned}
\frac{\mathrm{d} \mathbf{B}}{\mathrm{d} t} & =\mathbf{I} \dot{\mathbf{T}}_{\mathrm{IB}}=\mathbf{B} \mathbf{T}_{\mathrm{IB}}^{T} \cdot \dot{\mathbf{T}}_{\mathrm{IB}}, \\
\frac{\mathrm{d}^{2} \mathbf{B}}{\mathrm{d} t^{2}} & =\mathbf{B}\left(\mathbf{T}_{\mathrm{IB}}^{T} \dot{\mathbf{T}}_{\mathrm{IB}}\right)^{2}+\mathbf{B} \frac{\mathrm{d}}{\mathrm{d} t}\left(\mathbf{T}_{\mathrm{IB}}^{T} \cdot \dot{\mathbf{T}}_{\mathrm{IB}}\right) .
\end{aligned}
$$

The rotation angular velocity and acceleration of coordinate system $o x_{B} y_{B}$ relative to inertial reference system are, respectively, given as follows:

$$
\begin{aligned}
\vec{\omega} & =\vec{b}_{3}(\dot{\phi}-n), \\
\vec{\varepsilon} & =\vec{b}_{3} \ddot{\phi}
\end{aligned}
$$

where $\vec{b}_{3}=\vec{b}_{1} \times \vec{b}_{2}$. 
The position vector of the center of mass of link I relative to the origin of coordinate system $o x_{B} y_{B}$ is

$$
\vec{r}_{1 c}=\vec{d}+\frac{1}{2} \vec{l}_{1}
$$

where

$$
\begin{gathered}
\vec{d}=\mathbf{B}\left(\begin{array}{c}
-d \\
0
\end{array}\right), \\
\vec{l}_{1}=\mathbf{C}\left(\begin{array}{l}
l_{1} \\
0
\end{array}\right) .
\end{gathered}
$$

Substituting (14) into (13) meanwhile employing the third equation of (1) and the third equation of (3) yields

$$
\vec{r}_{1 c}=\mathbf{B}\left(\begin{array}{c}
-d-\frac{l_{1}}{2} \sin \theta_{1} \\
\frac{l_{1}}{2} \cos \theta_{1}
\end{array}\right) .
$$

Taking the first and second order time relative derivative of (15) yields the relative velocity and acceleration of the center of mass of link I with respect to coordinate system $o x_{B} y_{B}$ as follows:

$$
\begin{aligned}
& \vec{v}_{1 c}=\mathbf{B} \frac{\mathrm{d}}{\mathrm{d} t}\left(\begin{array}{c}
-d-\frac{l_{1}}{2} \sin \theta_{1} \\
\frac{l_{1}}{2} \cos \theta_{1}
\end{array}\right) . \\
& \vec{a}_{1 c}=\mathbf{B} \frac{\mathrm{d}^{2}}{\mathrm{~d} t^{2}}\left(\begin{array}{c}
-d-\frac{l_{1}}{2} \sin \theta_{1} \\
\frac{l_{1}}{2} \cos \theta_{1}
\end{array}\right) .
\end{aligned}
$$

(Note: the relative derivative of $\vec{r}_{1 c}$ does not contain the term $(\mathrm{d} \mathbf{B} / \mathrm{d} t)\left(\begin{array}{c}-d-\left(l_{1} / 2\right) \sin \theta_{1} \\ \left(l_{1} / 2\right) \cos \theta_{1}\end{array}\right)$. The same rule is suitable for the calculation of relative derivative of $\vec{v}_{1 c}$.)

The rotation angular velocity and acceleration of link I relative to coordinate system $o x_{B} y_{B}$ are, respectively, given as follows:

$$
\begin{aligned}
& \vec{\omega}_{1}=\vec{b}_{3} \dot{\theta}_{1}, \\
& \vec{\varepsilon}_{1}=\vec{b}_{3} \ddot{\theta}_{1} .
\end{aligned}
$$

The all forces upon link I (including the inertia forces) except for the constraint ones will be analyzed below.

The inertia force upon link I induced by linear acceleration $\vec{a}$ of coordinate system $o x_{B} y_{B}$ is

$$
\vec{F}_{1 a}=-m_{1} \vec{a} .
$$

The centrifugal force induced by rotation angular velocity $\vec{\omega}$ of coordinate system $o x_{B} y_{B}$ is

$$
\vec{F}_{1 \omega}=-m_{1} \vec{\omega} \times\left(\vec{\omega} \times \vec{r}_{1 c}\right) .
$$

The inertia force induced by rotation angular acceleration $\vec{\varepsilon}$ of coordinate system $o x_{B} y_{B}$ is

$$
\vec{F}_{1 \varepsilon}=-m_{1} \vec{\varepsilon} \times \vec{r}_{1 c} \text {. }
$$

The Coriolis force induced commonly by $\vec{\omega}$ and $\vec{v}_{1 c}$ is

$$
\vec{F}_{1 C}=-2 m_{1} \vec{\omega} \times \vec{v}_{1 c} .
$$

The relative inertia force induced by relative acceleration $\vec{a}_{1 c}$ of the center of mass of link I is

$$
\vec{F}_{1 a_{1 c}}=-m_{1} \vec{a}_{1 c} .
$$

All the moments of force (including the inertia forces) with respect to link I's center of mass will be analyzed below.

It can be proved that the moments of $\vec{F}_{1 a}, \vec{F}_{1 \omega}, \vec{F}_{1 C}$ are all zero (proof can be seen in Appendix). The moment of inertia force $\vec{F}_{1 \varepsilon}$ is

$$
\vec{M}_{1 \varepsilon}=-J_{1} \vec{\varepsilon}
$$

The moment of inertia force induced by relative angular acceleration $\vec{\varepsilon}_{1}$ of link I is

$$
\vec{M}_{1 \varepsilon_{1}}=-J_{1} \vec{\varepsilon}_{1} .
$$

Finally, the torque exerted on link I by the motors mounted on joints I and II is

$$
\vec{M}_{1}^{\prime}=\vec{b}_{3}\left(M_{1}+M_{2}\right) \text {. }
$$

The resultant force and moment exerted on link I are, respectively,

$$
\begin{aligned}
\vec{F}_{1} & =\vec{F}_{1 a}+\vec{F}_{1 \omega}+\vec{F}_{1 \varepsilon}+\vec{F}_{1 C}+\vec{F}_{1 a_{1 c}} \\
\vec{M}_{1 \text { total }} & =\vec{M}_{1 \varepsilon}+\vec{M}_{1 \varepsilon_{1}}+\vec{M}_{1}^{\prime} .
\end{aligned}
$$

Now we study link II.

The position vector of the center of mass of link II relative to the origin of coordinate system $o x_{B} y_{B}$ can be calculated out in the same way as for link I mentioned before:

$$
\vec{r}_{2 c}=\mathbf{B}\left(\begin{array}{c}
-d-l_{1} \sin \theta_{1}+\frac{1}{2} l_{2} \sin \left(\theta_{1}-\theta_{2}\right) \\
l_{1} \cos \theta_{1}-\frac{1}{2} l_{2} \cos \left(\theta_{1}-\theta_{2}\right)
\end{array}\right) .
$$

Taking the first and second order time relative derivative of (28) yields the relative velocity and acceleration of the center of mass of link II with respect to coordinate system $o x_{B} y_{B}$ as follows:

$$
\begin{aligned}
& \vec{v}_{2 c}=\mathbf{B} \frac{\mathrm{d}}{\mathrm{d} t}\left[\begin{array}{c}
-d-l_{1} \sin \theta_{1}+\frac{1}{2} l_{2} \sin \left(\theta_{1}-\theta_{2}\right) \\
l_{1} \cos \theta_{1}-\frac{1}{2} l_{2} \cos \left(\theta_{1}-\theta_{2}\right)
\end{array}\right] \\
& \vec{a}_{2 c}=\mathbf{B} \frac{\mathrm{d}^{2}}{\mathrm{~d} t^{2}}\left[\begin{array}{c}
-d-l_{1} \sin \theta_{1}+\frac{1}{2} l_{2} \sin \left(\theta_{1}-\theta_{2}\right) \\
l_{1} \cos \theta_{1}-\frac{1}{2} l_{2} \cos \left(\theta_{1}-\theta_{2}\right)
\end{array}\right] .
\end{aligned}
$$

The relative rotation angular velocity and acceleration of link II to coordinate system $o x_{B} y_{B}$ are, respectively,

$$
\begin{aligned}
& \vec{\omega}_{2}=\vec{b}_{3}\left(\dot{\theta}_{1}-\dot{\theta}_{2}\right), \\
& \vec{\varepsilon}_{2}=\vec{b}_{3}\left(\ddot{\theta}_{1}-\ddot{\theta}_{2}\right) .
\end{aligned}
$$


The inertia force upon link II induced by linear acceleration $\vec{a}$ of coordinate system $o x_{B} y_{B}$ is

$$
\vec{F}_{2 a}=-m_{2} \vec{a} .
$$

The centrifugal inertia force induced by rotation angular velocity $\vec{\omega}$ of coordinate system $o x_{B} y_{B}$ is

$$
\vec{F}_{2 \omega}=-m_{2} \vec{\omega} \times\left(\vec{\omega} \times \vec{r}_{2 c}\right) .
$$

The inertia force induced by rotation angular acceleration $\vec{\varepsilon}$ of coordinate system $o x_{B} y_{B}$ is

$$
\vec{F}_{2 \varepsilon}=-m_{2} \vec{\varepsilon} \times \vec{r}_{2 c} \text {. }
$$

The Coriolis force induced commonly by $\vec{\omega}$ and $\vec{v}_{2 c}$ is

$$
\vec{F}_{2 C}=-2 m_{2} \vec{\omega} \times \vec{v}_{2 c} .
$$

The relative inertia force induced by relative acceleration $\vec{a}_{2 c}$ of the center of mass of link II is

$$
\vec{F}_{2 a_{2 c}}=-m_{2} \vec{a}_{2 c} \text {. }
$$

All the moments of force (including the inertia forces) with respect to link II's center of mass will be analyzed below.

For the same reason as for link I aforementioned, among the moments of the inertia forces induced by the translational motion or rotation of coordinate system $o x_{B} y_{B}$, only the moment of inertia force $\vec{F}_{2 \varepsilon}$ needs to be calculated here as follows:

$$
\vec{M}_{2 \varepsilon}=-J_{2} \vec{\varepsilon}
$$

In addition, the moment of inertia force induced by relative angular acceleration $\vec{\varepsilon}_{2}$ of link II is

$$
\vec{M}_{2 \varepsilon_{2}}=-J_{2} \vec{\varepsilon}_{2}
$$

Finally, the torque exerted on link II by the motor mounted on joint II is

$$
\vec{M}_{2}^{\prime}=\vec{b}_{3}\left(-M_{2}\right)
$$

The resultant force and moment exerted on link II are, respectively,

$$
\begin{aligned}
\vec{F}_{2} & =\vec{F}_{2 a}+\vec{F}_{2 \omega}+\vec{F}_{2 \varepsilon}+\vec{F}_{2 C}+\vec{F}_{2 a_{2 c}} \\
\vec{M}_{2 \text { total }} & =\vec{M}_{2 \varepsilon}+\vec{M}_{2 \varepsilon_{2}}+\vec{M}_{2}^{\prime} .
\end{aligned}
$$

2.3. Equilibrium Equations of Generalized Forces. The essence of Kane method is to project all the active and inertia forces on the directions of the generalized coordinate curves, converting them into generalized forces, and then to write the equilibrium equations of the generalized forces. So it is also called generalized D'Alembert's principle. For the multibody system of two-link manipulator researched in the paper, rotation angles $\theta_{1}$ and $\theta_{2}$ of links I and II are defined as generalized coordinates, so the matrixes of projection (called partial velocity and/or partial angular velocity in Kane method) can be calculated out according to (15), (17), (28), and (30) as follows:

$$
\begin{aligned}
& \frac{\partial \vec{v}_{1 c}}{\partial \dot{\theta}_{1}}=\frac{\partial \vec{r}_{1 c}}{\partial \theta_{1}}=\mathbf{B}\left(\begin{array}{c}
-\frac{l_{1}}{2} \cos \theta_{1} \\
-\frac{l_{1}}{2} \sin \theta_{1}
\end{array}\right), \\
& \frac{\partial \vec{v}_{1 c}}{\partial \dot{\theta}_{2}}=\frac{\partial \vec{r}_{1 c}}{\partial \theta_{2}}=0, \\
& \frac{\partial \vec{v}_{2 c}}{\partial \dot{\theta}_{1}}=\frac{\partial \vec{r}_{2 c}}{\partial \theta_{1}}=\mathbf{B}\left(\begin{array}{l}
-l_{1} \cos \theta_{1}+\frac{1}{2} l_{2} \cos \left(\theta_{1}-\theta_{2}\right) \\
-l_{1} \sin \theta_{1}+\frac{1}{2} l_{2} \sin \left(\theta_{1}-\theta_{2}\right)
\end{array}\right), \\
& \frac{\partial \vec{v}_{2 c}}{\partial \dot{\theta}_{2}}=\frac{\partial \vec{r}_{2 c}}{\partial \theta_{2}}=\mathbf{B}\left(\begin{array}{l}
-\frac{1}{2} l_{2} \cos \left(\theta_{1}-\theta_{2}\right) \\
-\frac{1}{2} l_{2} \sin \left(\theta_{1}-\theta_{2}\right)
\end{array}\right), \\
& \frac{\partial \vec{\omega}_{1}}{\partial \dot{\theta}_{1}}=\vec{b}_{3}, \\
& \frac{\partial \vec{\omega}_{1}}{\partial \dot{\theta}_{2}}=0, \\
& \frac{\partial \vec{\omega}_{2}}{\partial \dot{\theta}_{1}}=\vec{b}_{3}, \\
& \vec{\theta}_{2} .
\end{aligned}
$$

The equilibrium equations of the generalized forces are

$$
\begin{aligned}
& \vec{F}_{1} \cdot \frac{\partial \vec{r}_{1 c}}{\partial \theta_{1}}+\vec{F}_{2} \cdot \frac{\partial \vec{r}_{2 c}}{\partial \theta_{1}}+\vec{M}_{1 \text { total }} \cdot \frac{\partial \vec{\omega}_{1}}{\partial \dot{\theta}_{1}}+\vec{M}_{2 \text { total }} \cdot \frac{\partial \vec{\omega}_{2}}{\partial \dot{\theta}_{1}} \\
& \quad=0 \\
& \vec{F}_{1} \cdot \frac{\partial \vec{r}_{1 c}}{\partial \theta_{2}}+\vec{F}_{2} \cdot \frac{\partial \vec{r}_{2 c}}{\partial \theta_{2}}+\vec{M}_{1 \text { total }} \cdot \frac{\partial \vec{\omega}_{1}}{\partial \dot{\theta}_{2}}+\vec{M}_{2 \text { total }} \cdot \frac{\partial \vec{\omega}_{2}}{\partial \dot{\theta}_{2}} \\
& \quad=0 .
\end{aligned}
$$

Substituting (5), (10) (12), (15) (16), (18) (29), and (31) (41) into (42) yields the system dynamics equations of the two-link manipulator as follows:

$$
\begin{aligned}
& -\left(J_{1}+J_{2}+\frac{1}{4} m_{1} l_{1}^{2}+m_{2} l_{1}^{2}+\frac{1}{4} m_{2} l_{2}^{2}\right. \\
& \left.-m_{2} l_{1} l_{2} \cos \theta_{2}\right) \ddot{\theta}_{1}+\left(J_{2}+\frac{1}{4} m_{2} l_{2}^{2}\right. \\
& \left.-\frac{1}{2} m_{2} l_{1} l_{2} \cos \theta_{2}\right) \ddot{\theta}_{2}+\left[\frac{1}{2} m_{1} l_{1} \cos \theta_{1}\right. \\
& \left.+m_{2} l_{1} \cos \theta_{1}-\frac{1}{2} m_{2} l_{2} \cos \left(\theta_{1}-\theta_{2}\right)\right](d-r) n^{2}
\end{aligned}
$$




$$
\begin{aligned}
& -\left[\left(m_{1}+2 m_{2}\right) l_{1} \sin \theta_{1}-m_{2} l_{2} \sin \left(\theta_{1}-\theta_{2}\right)\right] n \dot{r} \\
& -\left[m_{1} l_{1} \cos \theta_{1}+2 m_{2} l_{1} \cos \theta_{1}\right. \\
& \left.-m_{2} l_{2} \cos \left(\theta_{1}-\theta_{2}\right)\right](d-r) n \dot{\phi} \\
& +\left[\left(\frac{1}{2} m_{1}+m_{2}\right) l_{1} \cos \theta_{1}-\frac{1}{2} m_{2} l_{2} \cos \left(\theta_{1}-\theta_{2}\right)\right] \\
& \cdot \ddot{r}-\left[J_{1}+J_{2}+\frac{1}{4} m_{1} l_{1}^{2}+m_{2} l_{1}^{2}+\frac{1}{4} m_{2} l_{2}^{2}\right. \\
& +\left(\frac{1}{2} m_{1}+m_{2}\right)(d-r) l_{1} \sin \theta_{1} \\
& \left.-\frac{1}{2} m_{2}(d-r) l_{2} \sin \left(\theta_{1}-\theta_{2}\right)-m_{2} l_{1} l_{2} \cos \theta_{2}\right] \ddot{\phi} \\
& +\left[\left(\frac{1}{2} m_{1}+m_{2}\right) l_{1} \cos \theta_{1}-\frac{1}{2} m_{2} l_{2} \cos \left(\theta_{1}-\theta_{2}\right)\right] \\
& \cdot(d-r) \dot{\phi}^{2}+\left[\left(m_{1}+2 m_{2}\right) l_{1} \sin \theta_{1}\right. \\
& \left.-m_{2} l_{2} \sin \left(\theta_{1}-\theta_{2}\right)\right] \dot{r} \dot{\phi}+m_{2} l_{1} l_{2}(n-\dot{\phi}) \dot{\theta}_{2} \sin \theta_{2} \\
& +\frac{1}{2} m_{2} l_{1} l_{2} \dot{\theta}_{2}^{2} \sin \theta_{2}-m_{2} l_{1} l_{2} \dot{\theta}_{1} \dot{\theta}_{2} \sin \theta_{2}+M_{1} \\
& =0 \text {, } \\
& \left(J_{2}+\frac{1}{4} m_{2} l_{2}^{2}-\frac{1}{2} m_{2} l_{1} l_{2} \cos \theta_{2}\right) \ddot{\theta}_{1}-\left(J_{2}+\frac{1}{4} m_{2} l_{2}^{2}\right) \\
& \text {. } \ddot{\theta}_{2}+\frac{1}{2} m_{2}\left[(d-r) \cos \left(\theta_{1}-\theta_{2}\right)+l_{1} \sin \theta_{2}\right] l_{2} n^{2} \\
& -m_{2} l_{2} n \dot{r} \sin \left(\theta_{1}-\theta_{2}\right)-m_{2}\left[(d-r) \cos \left(\theta_{1}-\theta_{2}\right)\right. \\
& \left.+l_{1} \sin \theta_{2}\right] l_{2} n \dot{\phi}+\frac{1}{2} m_{2} l_{2} \ddot{r} \cos \left(\theta_{1}-\theta_{2}\right)+\left[J_{2}\right. \\
& +\frac{1}{4} m_{2} l_{2}^{2}+\frac{1}{2} m_{2}(r-d) l_{2} \sin \left(\theta_{1}-\theta_{2}\right) \\
& \left.-\frac{1}{2} m_{2} l_{1} l_{2} \cos \theta_{2}\right] \ddot{\phi}+\frac{1}{2} m_{2}\left[(d-r) \cos \left(\theta_{1}-\theta_{2}\right)\right. \\
& \left.+l_{1} \sin \theta_{2}\right] l_{2} \dot{\phi}^{2}+m_{2} l_{2} \dot{r} \dot{\phi} \sin \left(\theta_{1}-\theta_{2}\right) \\
& +m_{2} l_{1} l_{2}(\dot{\phi}-n) \dot{\theta}_{1} \sin \theta_{2}+\frac{1}{2} m_{2} l_{1} l_{2} \dot{\theta}_{1}^{2} \sin \theta_{2} \\
& +M_{2}=0 \text {. }
\end{aligned}
$$

\section{Design of the Control Torque of Joints}

The related information of space target such as its position relative to the carrier or the end-effector can be obtained easily by the camera mounted on the carrier-manipulator system [32-34]. The end-effector of the manipulator is programmed to move along a virtual line from the center of mass of the carrier to the space target until touch happens. At initial moment $(t=0)$, the manipulator is folded at $\theta_{1}=\theta_{10}$ and $\theta_{2}=\theta_{20}=2 \theta_{10}$, where $\theta_{10}$ is a positive small value. Assuming $l_{1}=l_{2}$, then the end-effector of the manipulator locates at a virtual line from the center of mass of the carrier to the space target, and the distance between them is $r-d-2 l_{1} \sin \theta_{10}$. At the initial moment, the manipulator is static relative to the carrier; that is, $\dot{\theta}_{1}=\dot{\theta}_{2}=0$; nevertheless, the endeffector moves toward the space target with the radial velocity $\dot{r}$ induced by the carrier's relative motion to the target. We set the time $t=t_{f}$ when the soft touch between the endeffector and the space target happens; that is, the distance and relative velocity between them reach zero simultaneously. We program the distance between the end-effector and the space target with respect to time as in the following polynomial function:

$$
\rho_{c}(t)=a_{0}+a_{1} t+a_{2} t^{2}+a_{3} t^{3}
$$

So

$$
\begin{aligned}
& \dot{\rho}_{c}(t)=a_{1}+2 a_{2} t+3 a_{3} t^{2} \\
& \ddot{\rho}_{c}(t)=2 a_{2}+6 a_{3} t .
\end{aligned}
$$

where $a_{i}(i=0 \sim 3)$ can be determined by the boundary conditions in time domain as follows:

$$
\begin{aligned}
\rho_{c}(0) & =a_{0}=r(0)-d-2 l_{1} \sin \theta_{10} \\
\dot{\rho}_{c}(0) & =a_{1}=\dot{r}(0) \\
\rho_{c}\left(t_{f}\right) & =a_{0}+a_{1} t_{f}+a_{2} t_{f}^{2}+a_{3} t_{f}^{3}=0 \\
\dot{\rho}_{c}\left(t_{f}\right) & =a_{1}+2 a_{2} t_{f}+3 a_{3} t_{f}^{2}=0 .
\end{aligned}
$$

The parameters $a_{i}(i=0 \sim 3)$ can be solved out easily according to (44a), (44b), (44c), and (45):

$$
\begin{aligned}
& a_{0}=r(0)-d-2 l_{1} \sin \theta_{10}, \\
& a_{1}=\dot{r}(0), \\
& a_{2}=-\frac{2 a_{1} t_{f}+3 a_{0}}{t_{f}^{2}}, \\
& a_{3}=\frac{a_{1} t_{f}+2 a_{0}}{t_{f}^{3}} .
\end{aligned}
$$

The command of the position of the end-effector under coordinate system $o x_{A} y_{A}$ should be as follows to meet the requirement aforementioned that the end-effector locates always at a virtual line from the center of mass of the carrier to the space target:

$$
\begin{aligned}
& x_{c}=\rho_{c} \cos \phi, \\
& y_{c}=\rho_{c} \sin \phi .
\end{aligned}
$$

The actual position coordinates of the end-effector are denoted by $\left(x_{e}, y_{e}\right)$, so we should demand

$$
\begin{aligned}
& x_{e}=x_{c}, \\
& y_{e}=y_{c} .
\end{aligned}
$$


The two-order time derivative of (48) is

$$
\begin{aligned}
& \ddot{x}_{e}=\ddot{x}_{c}, \\
& \ddot{y}_{e}=\ddot{y}_{c} .
\end{aligned}
$$

The position vector of the end-effector relative to the space target is

$$
\vec{r}_{e}=\mathbf{A}\left(\begin{array}{c}
x_{e} \\
y_{e}
\end{array}\right)=\vec{r}+\vec{d}+\vec{l}_{1}+\vec{l}_{2},
$$

where

$$
\vec{l}_{2}=\mathbf{D}\left(\begin{array}{l}
l_{2} \\
0
\end{array}\right) \text {. }
$$

Substituting (8), (14), and (51) into (50) meanwhile employing (1) and (3) yields the following:

$$
\begin{aligned}
x_{e}= & (r-d) \cos \phi-l_{1} \sin \left(\phi+\theta_{1}\right) \\
& +l_{2} \sin \left(\phi+\theta_{1}-\theta_{2}\right), \\
y_{e}= & (r-d) \sin \phi+l_{1} \cos \left(\phi+\theta_{1}\right) \\
& -l_{2} \cos \left(\phi+\theta_{1}-\theta_{2}\right) .
\end{aligned}
$$

Taking the second order derivative of (47) and (52) with respect to time, then substituting the results into (49) yields the following:

$$
\begin{aligned}
\ddot{\theta}_{1} & =\frac{1}{l_{1} \sin \theta_{2}}\left\{\left(\ddot{\rho}_{c}-\ddot{r}\right) \sin \left(\theta_{1}-\theta_{2}\right)-\left[l_{1} \sin \theta_{2}\right.\right. \\
& \left.+\left(d-r+\rho_{c}\right) \cos \left(\theta_{1}-\theta_{2}\right)\right] \ddot{\phi} \\
& +\left[\left(r-d-\rho_{c}\right) \sin \left(\theta_{1}-\theta_{2}\right)-l_{1} \cos \theta_{2}+l_{2}\right] \dot{\phi}^{2} \\
& +2\left(\dot{r}-\dot{\rho}_{c}\right) \dot{\phi} \cos \left(\theta_{1}-\theta_{2}\right)+2\left(l_{2}-l_{1} \cos \theta_{2}\right) \dot{\phi} \dot{\theta}_{1} \\
& \left.-2 l_{2} \dot{\phi}_{2}+\left(l_{2}-l_{1} \cos \theta_{2}\right) \dot{\theta}_{1}^{2}+l_{2} \dot{\theta}_{2}^{2}-2 l_{2} \dot{\theta}_{1} \dot{\theta}_{2}\right\} \\
\ddot{\theta}_{2} & =\frac{1}{l_{1} l_{2} \sin \theta_{2}}\left\{\left(\ddot{r}-\ddot{\rho}_{c}\right)\left[l_{1} \sin \theta_{1}-l_{2} \sin \left(\theta_{1}-\theta_{2}\right)\right]\right. \\
& +\ddot{\phi}\left(d-r+\rho_{c}\right)\left[l_{1} \cos \theta_{1}-l_{2} \cos \left(\theta_{1}-\theta_{2}\right)\right]+\left[l_{1}^{2}\right. \\
& +l_{2}^{2}+\left(d+\rho_{c}-r\right)\left(l_{1} \sin \theta_{1}-l_{2} \sin \left(\theta_{1}-\theta_{2}\right)\right) \\
& \left.-2 l_{1} l_{2} \cos \theta_{2}\right] \dot{\phi}^{2}+2\left(\dot{\rho}_{c}-r\right) \dot{\phi}\left[l_{1} \cos \theta_{1}\right. \\
& \left.-l_{2} \cos \left(\theta_{1}-\theta_{2}\right)\right]+2\left(l_{1}^{2}+l_{2}^{2}-2 l_{1} l_{2} \cos \theta_{2}\right) \dot{\phi} \dot{\theta}_{1} \\
& +2\left(l_{1} \cos \theta_{2}-l_{2}\right) l_{2} \dot{\phi}_{2}+\left(l_{1}^{2}+l_{2}^{2}-2 l_{1} l_{2} \cos \theta_{2}\right) \\
& . \dot{\theta}_{1}^{2}+\left(l_{2}-l_{1} \cos \theta_{2}\right) l_{2} \dot{\theta}_{2}^{2}+2\left(l_{1} \cos \theta_{2}-l_{2}\right) \\
& \left.l_{2} \dot{\theta}_{1} \dot{\theta}_{2}\right\} \cdot
\end{aligned}
$$

TABLE 1: Inlet parameters for numerical simulation.

\begin{tabular}{lc}
\hline Inlet parameters & Values \\
\hline$m_{1}(\mathrm{~kg})$ & 2 \\
$m_{2}(\mathrm{~kg})$ & 2 \\
$l_{1}(\mathrm{~m})$ & 6 \\
$l_{2}(\mathrm{~m})$ & 6 \\
$J_{1 c}\left(\mathrm{~kg} \cdot \mathrm{m}^{2}\right)$ & 6 \\
$J_{2 c}\left(\mathrm{~kg} \cdot \mathrm{m}^{2}\right)$ & 6 \\
$d(\mathrm{~m})$ & 2 \\
$n(\mathrm{rad} / \mathrm{s})$ & 0.0012 \\
$x_{0}(\mathrm{~m})$ & 10 \\
$y_{0}(\mathrm{~m})$ & 2 \\
$\theta_{10}(\mathrm{deg})$ & 5 \\
$\theta_{20}(\mathrm{deg})$ & 10 \\
$t_{f}(\mathrm{~s})$ & 60 \\
\hline
\end{tabular}

$\ddot{\theta}_{1}, \ddot{\theta}_{2}$ shown in (53) are the demanded rotation angular acceleration of links I and II, respectively, for that the endeffector of the manipulator can move in our programmed path aforementioned and touch the target softly at final time $t_{f}$.

Substituting (6a), (6b), (6c), (7a), (7b), (7c), (44a), (44b), (44c), (46), and (53) into (43a) and (43b) can yield the demanded driving torques $M_{1}$ and $M_{2}$ expressed as the functions of the current time $t$, the current rotation angle, and angular velocity of the two links I and II $\theta_{1}, \theta_{2}, \dot{\theta}_{1}, \dot{\theta}_{2}$, with $x_{0}, y_{0}$ as parametric variables. That is to say, $M_{i}=$ $M_{i}\left(t, \theta_{1}, \theta_{2}, \dot{\theta}_{1}, \dot{\theta}_{2} ; x_{0}, y_{0}\right)(i=1,2)$. Due to space limitations, the detailed expressions of them will not be written here.

It can be noticed that (53) will be singular if $\theta_{2}=0$. So the manipulator will not be fully folded (i.e., $\theta_{10}=$ $\theta_{20}=0$ ) at initial time of deployment procedure in order to avoid singularity. $\theta_{10}$ takes a small positive value $\varepsilon$, and $\theta_{20}=2 \theta_{10}$ so as to guarantee the end-effector locates at a virtual line connecting the carrier and the space target at initial time; then driven by the open loop control law $M_{i}=$ $M_{i}\left(t, \theta_{1}, \theta_{2}, \dot{\theta}_{1}, \dot{\theta}_{2} ; x_{0}, y_{0}\right)(i=1,2)$ designed before, $\theta_{2}$ will increase gradually, but it will not reach $\pi$ until touching the space target since the total length of the two links I and II is bigger than the distance between the carrier and the target in the cases we study. So the initial value of $\theta_{10}=\varepsilon>0$ and $\theta_{20}=2 \theta_{10}$ can avoid the dynamics singularity during approaching operation.

\section{Numerical Simulation}

The inlet parameters for numerical simulation are shown in Table 1 .

The results of numerical simulation are shown in Figures 2 7.

The results of the numerical simulation indicate that the actual position of the end-effector closely tracks the command; in fact, the response curves almost overlap the command curves exactly. At the final time $t=t_{f}=60$ (s) we preassigned, the distance and the radial velocity of the endeffector relative to the space target reach zero simultaneously, 


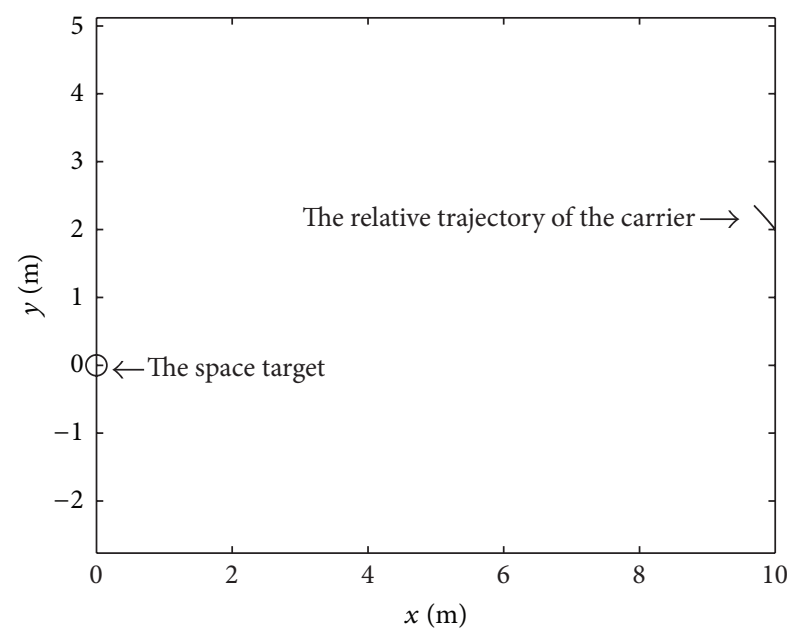

FIgURE 2: The relative trajectory of the carrier to the space target.

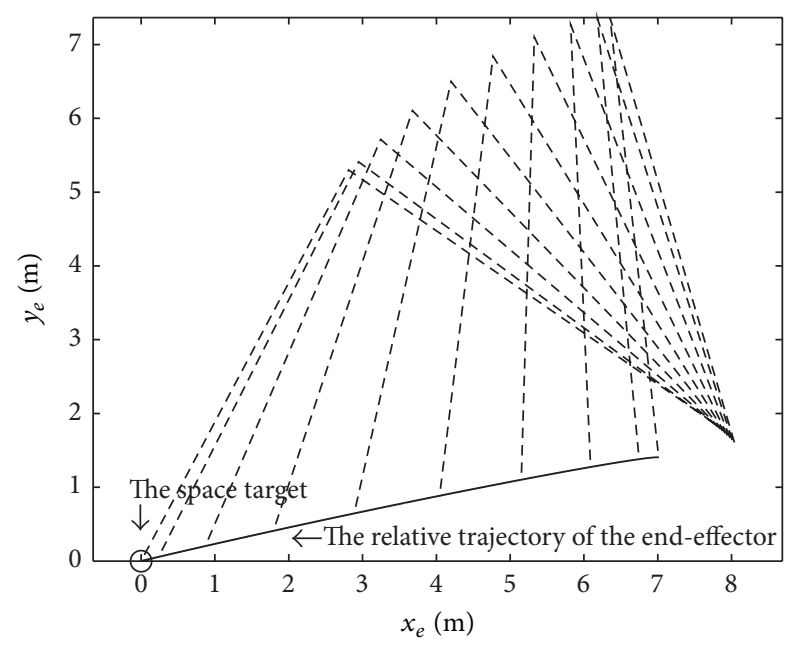

FIGURE 3: The relative trajectory of the end-effector to the space target and the corresponding configuration variation of the manipulator.

satisfying the requirement of soft touch, so avoiding the probable damage to the end-effector or the space target by the impact between them. We also see from the simulation results that the driving torques of the motors are less than $0.1(\mathrm{~N} \cdot \mathrm{m})$, so it is easy to implement in engineering practice.

The simulation neglecting the inertia forces exerted on the manipulators induced by the carrier body-fixed coordinate system has been conducted as well. The deviations of the simulation results neglecting the inertia forces and moments from that considering the inertia forces and moments mentioned above have been shown in Figures 8 10.

In Figures 8 10, the variables labeled by “*” such as $M_{1}^{*}$ $\rho_{e}^{*}$ come from the simulation neglecting the inertia forces exerted on the space manipulators. From the third curve of Figure 10, we see that the deviation of the distance between the end-effector and the space target reaches up to $0.15(\mathrm{~m})$ at final time $t_{f}=60(\mathrm{~s})$ from the simulation results considering the inertia forces. Since $\rho_{e}\left(t_{f}\right)=0$, then $\rho_{e}^{*}\left(t_{f}\right)=\rho_{e}\left(t_{f}\right)+$
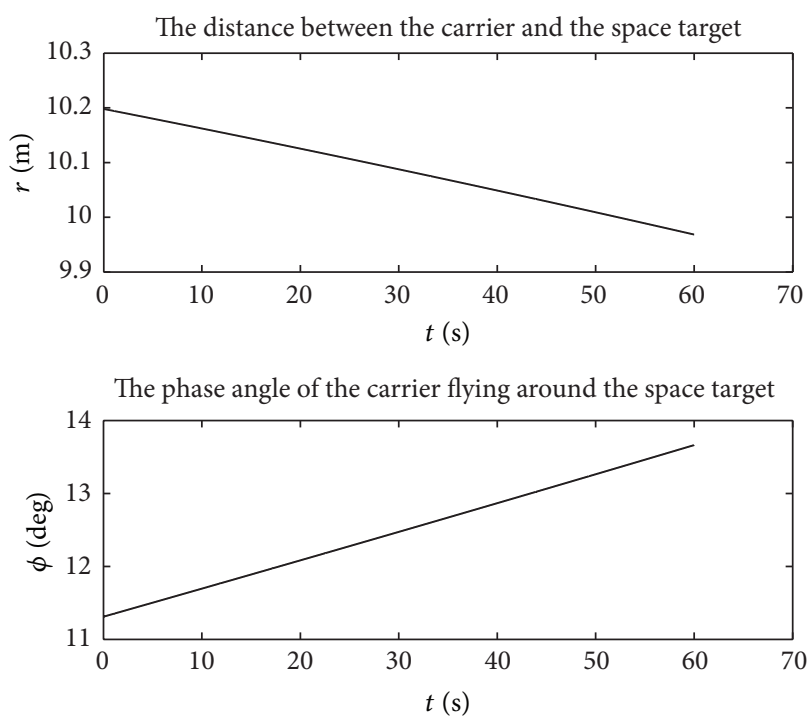

FIGURE 4: The distance and phase angle of the carrier relative to the space target.
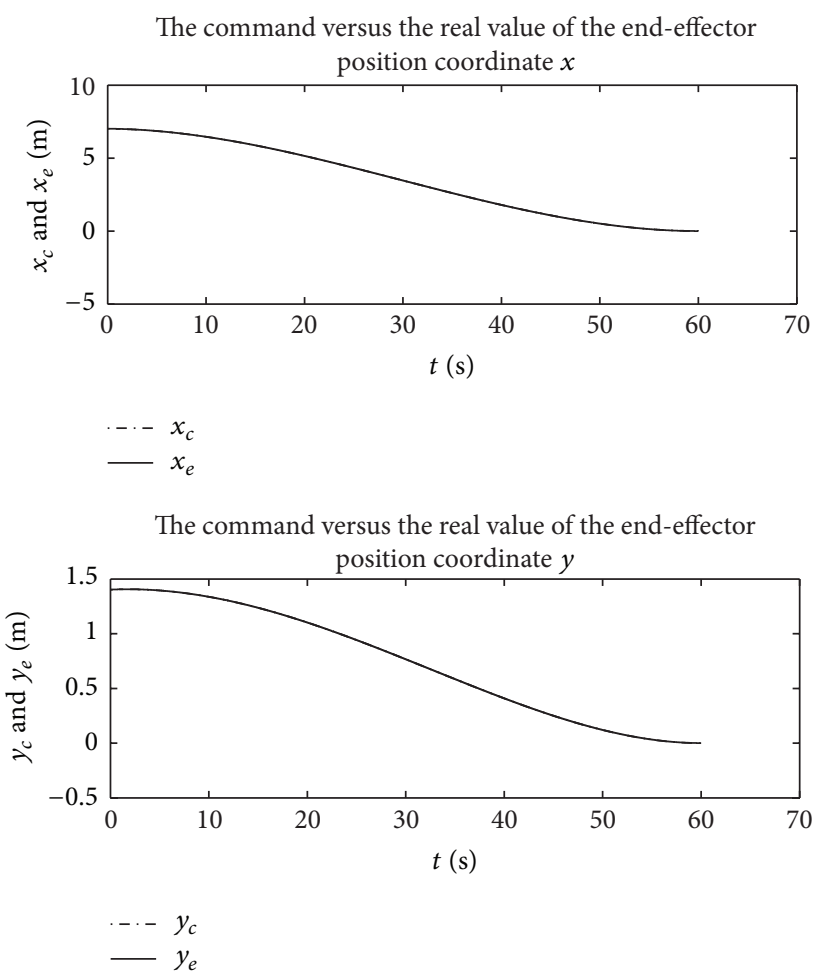

FIgURE 5: The command versus the real value of the end-effector position coordinate.

$\Delta \rho_{e}\left(t_{f}\right)=0.15(\mathrm{~m})$. The miss distance of $15(\mathrm{~cm})$ is too big to realize pinpoint capturing target for on-orbit operation. In the future, more quick and more pinpoint operation of space manipulator may be required, and then the rotation angular velocity of the manipulator's links will be faster than that in the simulation here. In that situation, the inertia force such as Coriolis force induced commonly by the coordinate 


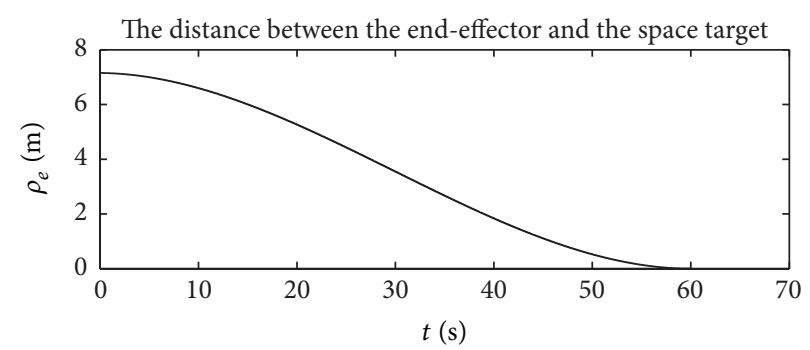

The radial velocity of the end-effector relative to

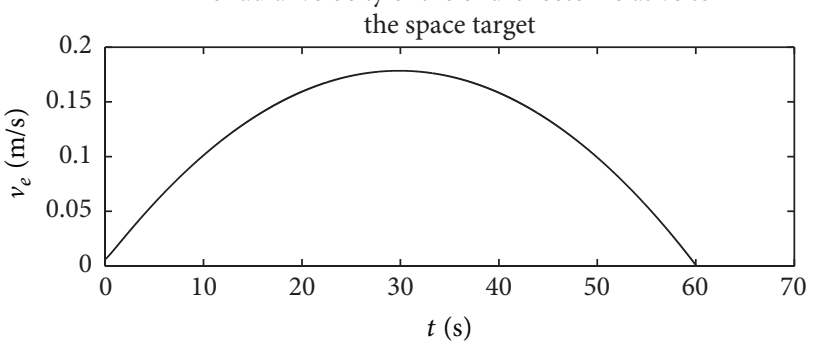

FIGURE 6: The distance and relative velocity of the end-effector to the space target.

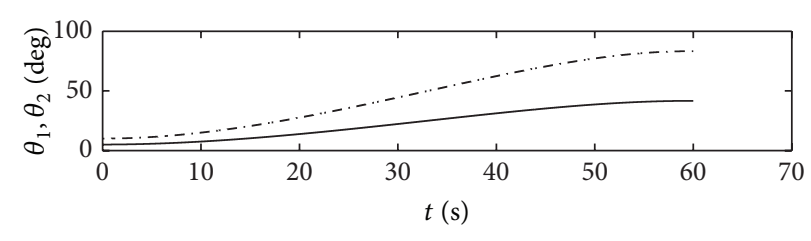

$$
\begin{array}{r}
\theta_{1} \\
--\theta_{2}
\end{array}
$$

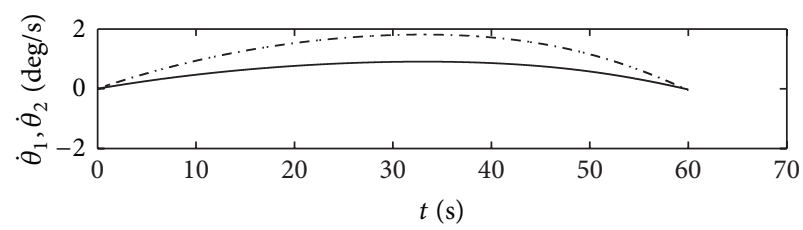

$$
-\dot{\theta}_{1}
$$$$
-.-\dot{\theta}_{2}
$$

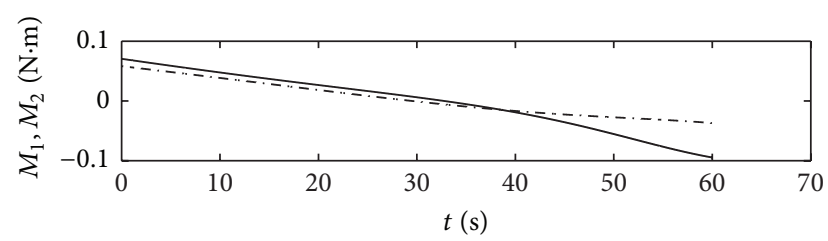

$$
\begin{array}{r}
-M_{1} \\
--M_{2}
\end{array}
$$

Figure 7: The rotation angles, angular velocities of the links I and II, and the corresponding driving torques of the motors.

system's rotation and the manipulator's links relative motion will be larger than that in the simulation here, so they cannot be neglected even more in dynamics modeling and control design.
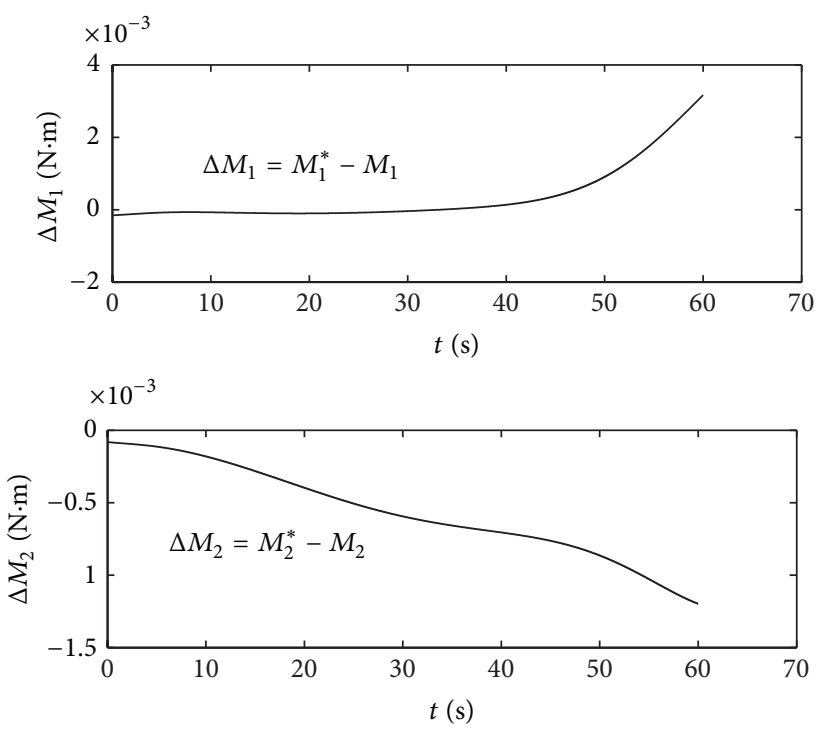

FIGURE 8: The deviation of driving torques neglecting the inertia forces and moments from that considering them.
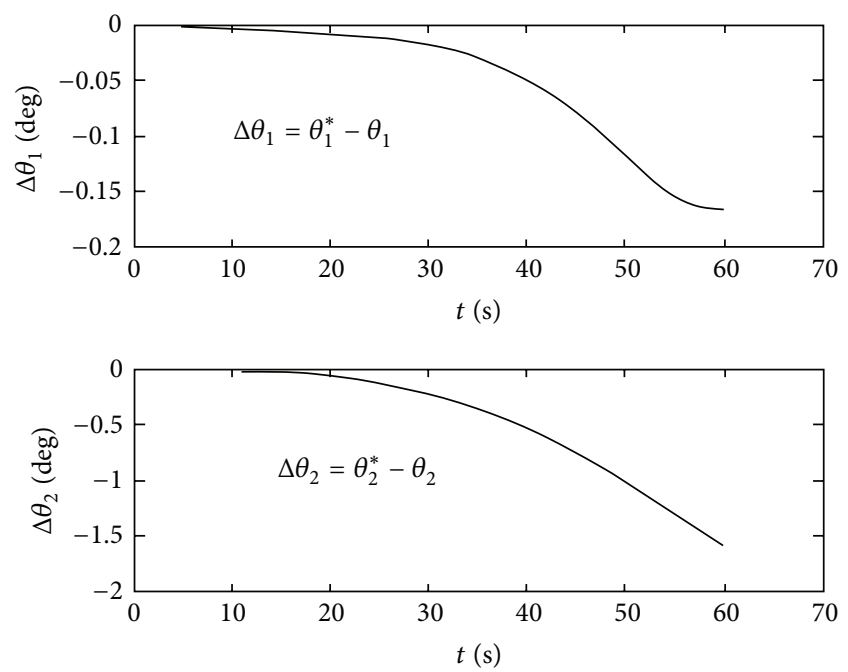

Figure 9: The deviation of link's rotation angles neglecting the inertia forces and moments from that considering them.

\section{Conclusions}

The two-link manipulator's dynamics model during its endeffector approaching of a space target is derived using Kane method under the situation that the carrier is flying freely around the space target, governed by the C-W equations. The relative orbit dynamics of the carrier to the space target is considered in system dynamics modeling. The path functions of the end-effector of the manipulator as well as the corresponding driving torques functions of the joints are programmed in accordance with the requirement of soft touch between the end-effector and the target for the purpose of avoiding the damage to them by touch impact. The results of numerical simulation show the effectiveness of the control law proposed in the paper. The issues left for 

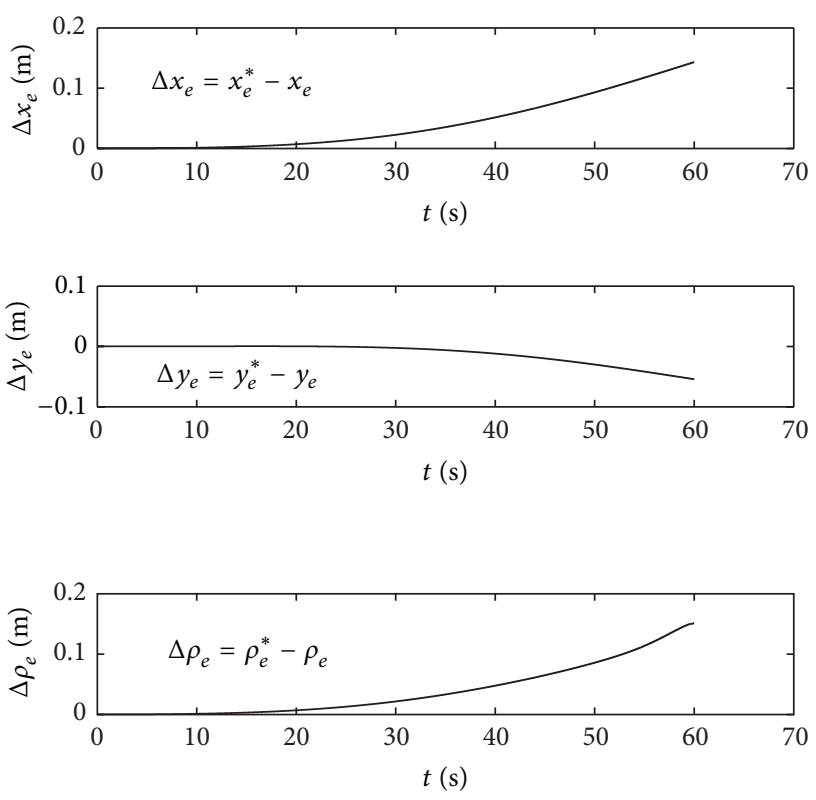

FIgURE 10: The deviation of end-effector's position coordinates and its distance relative to the space target neglecting the inertia forces and moments from that considering them.

the future study are as follows: (1) the coupling dynamics between the carrier and the manipulators; (2) the complicated manipulator system dynamics in the nonideal situations such that the orbit of the space target around the earth is not circle with $\mathrm{C}-\mathrm{W}$ equations being not tenable; and (3) the optimization of the open control law for the driving torque or the design of closed loop control law so as to acquire the robustness to perturbations.

\section{Appendix}

As shown in Figure 11, coordinate system $o x_{B} y_{B}$ is noninertial. Specifically speaking, linear acceleration $\vec{a}$ of its origin $o$ and angular velocity $\vec{\omega}$ (perpendicular to the page) of the coordinate system are not zero. $o^{\prime}$ is the center of mass of a rigid body under the coordinate system, and its position vector relative to point $o$ is $\vec{r}_{0} . \vec{v}_{0} \triangleq \dot{\vec{r}}_{0}$ is the velocity vector of $o^{\prime}$ relative to point $o$. The rotation angular velocity of the rigid body relative to coordinate system $o x_{B} y_{B}$ is $\vec{\Omega}$ (perpendicular to the page). $\mathrm{d} m$ is a mass infinitesimal of the rigid body, and the position vector of it relative to point $o^{\prime}$ is $\vec{r}$. Now we will prove that the moments of the inertia forces induced by $\vec{a}, \vec{\omega}$ with respect to the center of mass of the rigid body are all zero in two-dimension situation.

The inertia force induced by acceleration $\vec{a}$ exerted on the mass infinitesimal $\mathrm{d} m$ of the rigid body is

$$
\mathrm{d} \vec{F}_{a}=-\vec{a} \mathrm{~d} m .
$$

The moment of the infinitesimal force with respect to point $o^{\prime}$ is

$$
\mathrm{d} \vec{M}_{a}=\vec{r} \times \mathrm{d} \vec{F}_{a}
$$

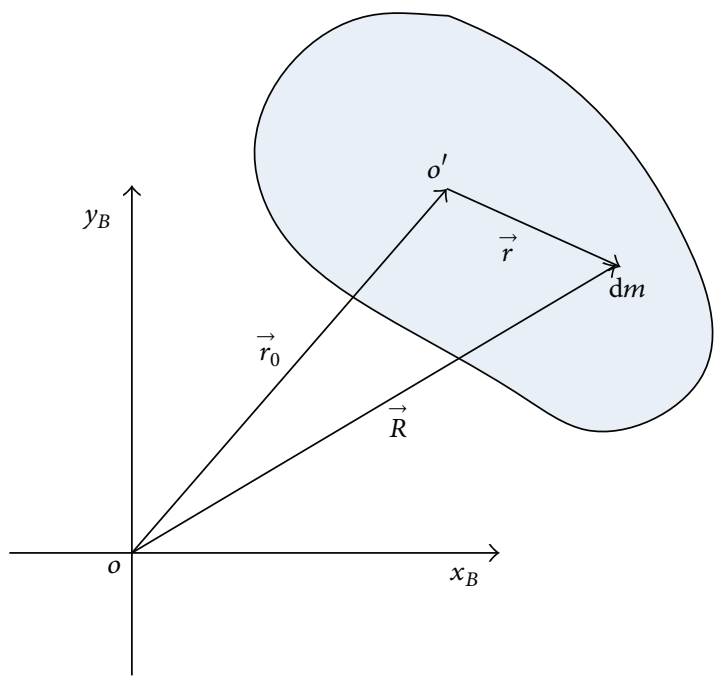

FIGURE 11: Illustration of a rigid body in noninertial coordinate system.

The corresponding total moment with respect to point $o^{\prime}$ is obviously

$$
\vec{M}_{a}=\int \mathrm{d} \vec{M}_{a} .
$$

Substituting (A.1) and (A.2) into (A.3), we obtain

$$
\begin{aligned}
\vec{M}_{a} & =\int \mathrm{d} \vec{M}_{a}=\int \vec{r} \times(-\vec{a} \mathrm{~d} m)=-\int \vec{r} \mathrm{~d} m \times \vec{a} \\
& =0 \times \vec{a}=0 .
\end{aligned}
$$

That is,

$$
\vec{M}_{a}=0 .
$$

The definition of the center of mass of rigid body is used in the above derivation.

The inertia centrifugal force induced by angular velocity $\vec{\omega}$ exerted on mass infinitesimal $\mathrm{d} m$ is

$$
\mathrm{d} \vec{F}_{\omega}=-\vec{\omega} \times(\vec{\omega} \times \vec{R}) \mathrm{d} m,
$$

where

$$
\vec{R}=\vec{r}_{0}+\vec{r} .
$$

The moment of the infinitesimal force with respect to point $o^{\prime}$ is

$$
\mathrm{d} \vec{M}_{\omega}=\vec{r} \times \mathrm{d} \vec{F}_{\omega} .
$$

The corresponding total moment with respect to point $o^{\prime}$ is obviously

$$
\vec{M}_{\omega}=\int \mathrm{d} \vec{M}_{\omega}
$$


Substituting (A.6) (A.8) into (A.9), we obtain

$$
\begin{aligned}
\vec{M}_{\omega} & =\int \mathrm{d} \vec{M}_{\omega}=\int \vec{r} \times[-\vec{\omega} \times(\vec{\omega} \times \vec{R}) \mathrm{d} m] \\
& =-\int \vec{r} \times[\vec{\omega} \times(\vec{\omega} \times \vec{R})] \mathrm{d} m \\
& =-\int \vec{r} \times[(\vec{\omega} \cdot \vec{R}) \vec{\omega}-(\vec{\omega} \cdot \vec{\omega}) \vec{R}] \mathrm{d} m \\
& =-\int \vec{r} \times[0 \vec{\omega}-(\vec{\omega} \cdot \vec{\omega}) \vec{R}] \mathrm{d} m \\
& =\int \vec{r} \times[(\vec{\omega} \cdot \vec{\omega}) \vec{R}] \mathrm{d} m=(\vec{\omega} \cdot \vec{\omega}) \int \vec{r} \times \vec{R} \mathrm{~d} m \\
& =(\vec{\omega} \cdot \vec{\omega}) \int \vec{r} \times\left(\vec{r}_{0}+\vec{r}\right) \mathrm{d} m \\
& =(\vec{\omega} \cdot \vec{\omega}) \int\left(\vec{r} \times \vec{r}_{0}+\vec{r} \times \vec{r}\right) \mathrm{d} m \\
& =(\vec{\omega} \cdot \vec{\omega}) \int\left(\vec{r} \times \vec{r}_{0}+0\right) \mathrm{d} m \\
& =(\vec{\omega} \cdot \vec{\omega}) \int \vec{r} \times \vec{r}_{0} \mathrm{~d} m=(\vec{\omega} \cdot \vec{\omega})\left(\int \vec{r} \mathrm{~d} m\right) \times \vec{r}_{0} \\
& (\vec{\omega}) 0 \times \vec{r}_{0}=0 .
\end{aligned}
$$

That is,

$$
\vec{M}_{\omega}=0
$$

The Coriolis force induced commonly by angular velocity $\vec{\omega}$ and relative motion $\dot{\vec{R}}$ of $\mathrm{d} m$ to point $o$ exerted on mass infinitesimal $\mathrm{d} m$ is

$$
\mathrm{d} \vec{F}_{C}=-2 \vec{\omega} \times \dot{\vec{R}} \mathrm{~d} m
$$

where $\dot{\vec{R}}$ denotes the time relative derivative of $\vec{R}$ with respect to coordinate system $o x_{B} y_{B}$.

The moment of the infinitesimal force with respect to point $o^{\prime}$ is

$$
\mathrm{d} \vec{M}_{C}=\vec{r} \times \mathrm{d} \vec{F}_{C}
$$

The corresponding total moment with respect to point $o^{\prime}$ is obviously

$$
\vec{M}_{C}=\int \mathrm{d} \vec{M}_{C}
$$

Substituting (A.12) and (A.13) into (A.14), we obtain

$$
\begin{aligned}
\vec{M}_{C} & =\int \mathrm{d} \vec{M}_{C}=\int \vec{r} \times \mathrm{d} \vec{F}_{C}=\int \vec{r} \times(-2 \vec{\omega} \times \dot{\vec{R}} \mathrm{~d} m) \\
& =-2 \int \vec{r} \times(\vec{\omega} \times \dot{\vec{R}}) \mathrm{d} m \\
& =-2 \int[(\vec{r} \cdot \dot{\vec{R}}) \vec{\omega}-(\vec{r} \cdot \vec{\omega}) \dot{\vec{R}}] \mathrm{d} m
\end{aligned}
$$

$$
\begin{aligned}
& =-2 \int[(\vec{r} \cdot \dot{\vec{R}}) \vec{\omega}-0 \dot{\vec{R}}] \mathrm{d} m \\
& =-2 \int(\vec{r} \cdot \dot{\vec{R}}) \vec{\omega} \mathrm{d} m=-2 \vec{\omega} \int(\vec{r} \cdot \dot{\vec{R}}) \mathrm{d} m \\
& =-2 \vec{\omega} \int\left[\vec{r} \cdot\left(\dot{\vec{r}}_{0}+\dot{\vec{r}}\right)\right] \mathrm{d} m \\
& =-2 \vec{\omega}\left(\int \vec{r} \cdot \dot{\vec{r}}_{0} \mathrm{~d} m+\int \vec{r} \cdot \dot{\vec{r}} \mathrm{~d} m\right) \\
& =-2 \vec{\omega}\left[\int \vec{r} \mathrm{~d} m \cdot \dot{\vec{r}}_{0}+\int \vec{r} \cdot(\vec{\Omega} \times \vec{r}) \mathrm{d} m\right] \\
& =-2 \vec{\omega}\left(0 \cdot \dot{\vec{r}}_{0}+\int 0 \mathrm{~d} m\right)=0 .
\end{aligned}
$$

That is,

$$
\vec{M}_{C}=0 \text {. }
$$

In summary, we conclude that in two-dimensional situations, namely, planar situations, the inertia force moments exerted on a rigid body with respect to its center of mass induced by the coordinate system's linear acceleration, centripetal acceleration, and the rigid body's Coriolis acceleration are all zero.

\section{Competing Interests}

The author declares that there is no conflict of interests regarding the publication of this paper.

\section{Acknowledgments}

This work is supported by the Fundamental Research Funds for the Central Universities (Grant no. NS2016082).

\section{References}

[1] K. D. Kumar, "Review of dynamics and control of nonelectrodynamic tethered satellite systems," Journal of Spacecraft and Rockets, vol. 43, no. 4, pp. 705-720, 2006.

[2] L. Hong, J. Zai-Nan, and L. Ye-Chao, "Review of space manipulator technology," Manned Spaceflight, vol. 21, no. 5, pp. 435-443, 2015.

[3] L. Daming, R. Wei, H. Chengwei, W. Yaobing, T. Zixin, and W. Youyu, "Overview of the Chinese space station manipulator," in Proceedings of the AIAA SPACE 2015 Conference and Exposition, SPACE Conferences and Exposition, Pasadena, Calif, USA, 2015.

[4] M. A. Torres and S. Dubowsky, "Minimizing spacecraft attitude disturbances in space manipulator systems," Journal of Guidance, Control, and Dynamics, vol. 15, no. 4, pp. 1010-1017, 1992.

[5] F. Aghili, "Cartesian control of space manipulators for on-orbit servicing," in Proceedings of the AIAA Guidance, Navigation and Control Conference and Exhibit, Toronto, Canada, August 2010.

[6] P. J. From, K. Y. Pettersen, and J. T. Gravdahl, "A singular free formulation of the dynamically equivalent manipulator mapping of space manipulators," in Proceedings of the AIAA SPACE Conference \& Exposition, Long Beach, Calif, USA, 2011. 
[7] P. Trivailo and T. Gilbert, "Dynamic modelling of flexible space robotic manipulators during payload capture," in Proceedings of the 55th International Astronautical Congress, pp. 433-444, Vancouver, Canada, October 2004.

[8] O. Ma, T. Chen, and Z. Zhao, "Understanding the dynamics of a space manipulator from its testing with air-bearing based support equipment," in Proceedings of the SPACE Conference and Exposition, Pasadena, Calif, USA, 2015.

[9] E. E. Komendera, W. R. Doggett, and J. T. Dorsey, "Preliminary demonstration for a tendon-actuated lightweight in-space manipulator," in Proceedings of the AIAA Space Conference and Exposition, Pasadena, Calif, USA, 2015.

[10] C. Altenbuchner, J. Dorsey, and T. C. Jones, "Flexible multibody dynamics modeling of a tendon-actuated lightweight inspace manipulator," in Proceedings of the AIAA SPACE 2015 Conference and Exposition, SPACE Conferences and Exposition, Pasadena, Calif, USA, 2015.

[11] W. R. Doggett, J. T. Dorsey, T. C. Jones et al., "Improvements to the Tendon-Actuated Lightweight In-Space MANipulator (TALISMAN) system," in Proceedings of the SPACE Conference and Exposition, Pasadena, Calif, USA, 2015.

[12] K. Senda, "Quasioptimal control of space redundant manipulators," in Proceedings of the Guidance, Navigation, and Control Conference and Exhibit, Guidance, Navigation, and Control and Co-located Conferences, AIAA-99-4303:1877-1885, 1999.

[13] Q.-X. Jia, L. Zhang, G. Chen, and H.-X. Sun, "Pre-impact trajectory optimization of redundant space manipulator with multi-target fusion," Journal of Astronautics, vol. 35, no. 6, pp. 639-647, 2014.

[14] T. Rybus, K. Seweryn, and J. Z. Sąsiadek, “Trajectory optimization of space manipulator with non-zero angular momentum during orbital capture maneuver," in Proceedings of the AIAA Guidance, Navigation, and Control Conference, San Diego, Calif, USA, January 2016.

[15] Y. Matsuzaki and T. Hayatoma, "Positioning control of a flexible manipulator of a space robot based on artificial potential method," Tech. Rep. AIAA-98-2094-CP:3279-3292, 1998.

[16] S. Ueno and V. J. Modi, "Optimal controller for a space platform based manipulator capturing a satellite," in Proceedings of the AIAA/AAS Astrodynamics Specialist Conference and Exhibit, pp. 324-338, American Institute of Aeronautics and Astronautics Inc, AIAA, San Diego, Calif, USA, 1998.

[17] G. Gilardi, S. Kawamoto, and S. Kibe, "Capture of a noncooperative object using a two-arm manipulator," in Proceedings of the 55th International Astronautical Congress, Vancouver, Canada, October 2004.

[18] K. Yoshida, K. Hashizume, D. N. Nenchev, N. Inaba, and M. Oda, "Control of a space manipulator for autonomous target capture-ETS-VII flight experiments and analysis," in Proceedings of the AIAA Guidance, Navigation, and Control Conference and Exhibit, Denver, Colo, USA, August 2000.

[19] M. J. Sadigh and A. Salehi, "A robust closed loop control for fast maneuvers of micro-macro manipulators," in Proceedings of the 55th International Astronautical Congress, Vancouver, Canada, 2004.

[20] J. F. Goulet, C. W. De Silva, V. J. Modi, and A. K. Misra, "Hierarchical control of a space-based deployable manipulator using fuzzy logic," Journal of Guidance, Control, and Dynamics, vol. 24, no. 2, pp. 395-405, 2001.

[21] H. Fujii and S. Ishijima, "Mission function control for deployment and retrieval of a subsatellite," Journal of Guidance, Control, and Dynamics, vol. 12, no. 2, pp. 243-247, 1989.
[22] H. Fujii, K. Uchiyama, and K. Kokubun, "Mission function control of tethered subsatellite deployment/retrieval: in-plane and out-of-plane motion," Journal of Guidance, Control, and Dynamics, vol. 14, no. 2, pp. 471-473, 1991.

[23] H. A. Fujii and S. Anazawa, "Deployment/retrieval control of tethered subsatellite through an optimal path," Journal of Guidance, Control, and Dynamics, vol. 17, no. 6, pp. 1292-1298, 1994.

[24] K. Kokubun and H. A. Fujii, "Deployment/retrieval control of a tethered subsatellite under effect of tether elasticity," Journal of Guidance, Control, and Dynamics, vol. 19, no. 1, pp. 84-90, 1996.

[25] K. Kokubun, S. Anazawa, and H. A. Fujii, "Real-time optimal state feedback control for tethered subsatellite system," Journal of Guidance, Control, and Dynamics, vol. 19, no. 4, pp. 972-974, 1996.

[26] S. Yu, "Periodic motion in the tethered satellite system," Journal of Guidance, Control, and Dynamics, vol. 19, no. 5, pp. 1195-1197, 1996.

[27] H. A. Fujii, W. Ichiki, S.-I. Suda, and T. R. Watanabe, "Chaos analysis on librational control of gravity-gradient satellite in elliptic orbit," Journal of Guidance, Control, and Dynamics, vol. 23, no. 1, pp. 145-146, 2000.

[28] N. Takeichi, M. C. Natori, and N. Okuizumi, "Fundamental strategies for control of a tethered system in elliptical orbits," Journal of Spacecraft and Rockets, vol. 40, no. 1, pp. 119-125, 2003.

[29] H. Kojima, M. Iwasaki, H. A. Fujii, C. Blanksby, and P. Trivailo, "Nonlinear control of librational motion of tethered satellites in elliptic orbits," Journal of Guidance, Control, and Dynamics, vol. 27, no. 2, pp. 229-239, 2004.

[30] H. Kojima, Y. Furukawa, and P. M. Trivailo, "Experimental verification of periodic libration of tethered satellite system in elliptic orbit," Journal of Guidance, Control, and Dynamics, vol. 34, no. 2, pp. 614-618, 2011.

[31] X. Xiao-Ning, W. Wei, and G. Yu-Dong, The Basis of Orbit Dynamics for Near-Earth Spacecraft, National University of Defense Technology Press, Changsha, China, 2003.

[32] Q.-M. Tan, C.-W. Hu, and S. Gao, "Research on calibration of intrinsic parameters for space manipulator camera based on 2D planar pattern," Spacecraft Recovery and Remote Sensing, vol. 34, no. 6, pp. 74-80, 2013.

[33] L. Yu, C. Feng, H. Jian-Min et al., "Study on vision measurement technology of space robotic arm," Manned Spaceflight, vol. 20, no. 2, pp. 127-133, 2014.

[34] G. Dong and Z. H. Zhu, "Vision-based pose and motion estimation of non-cooperative target for space robotic manipulators," in Proceedings of the AIAA Space Conference and Exposition, San Diego, Calif, USA, August 2014. 


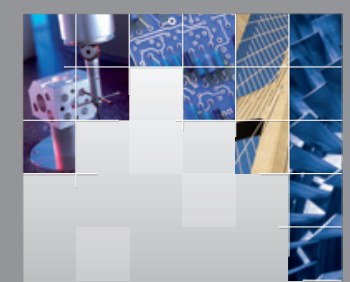

\section{Enfincering}
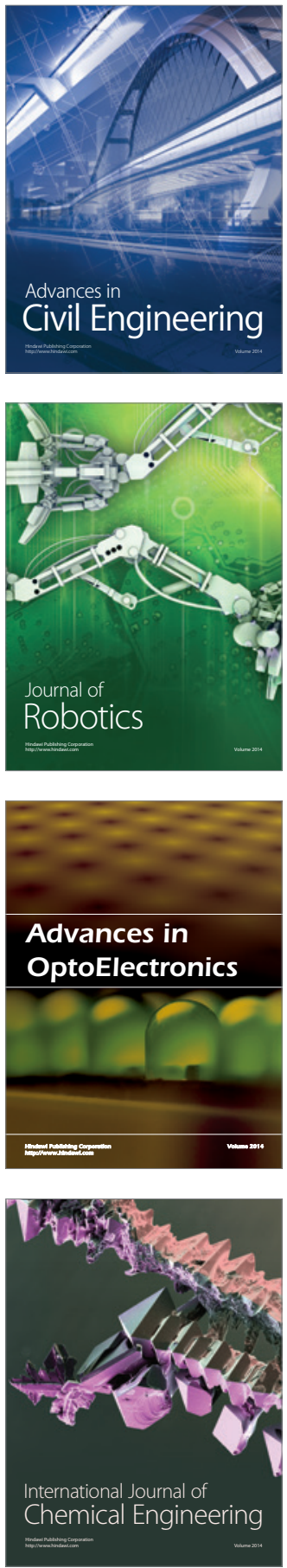

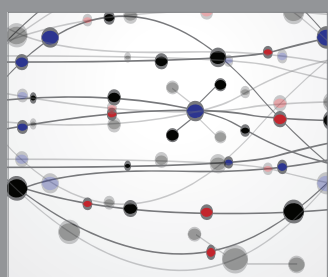

The Scientific World Journal

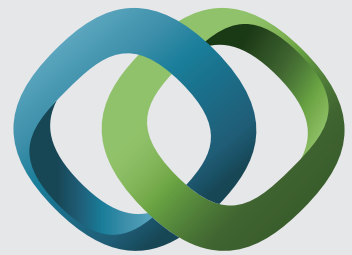

\section{Hindawi}

Submit your manuscripts at

http://www.hindawi.com
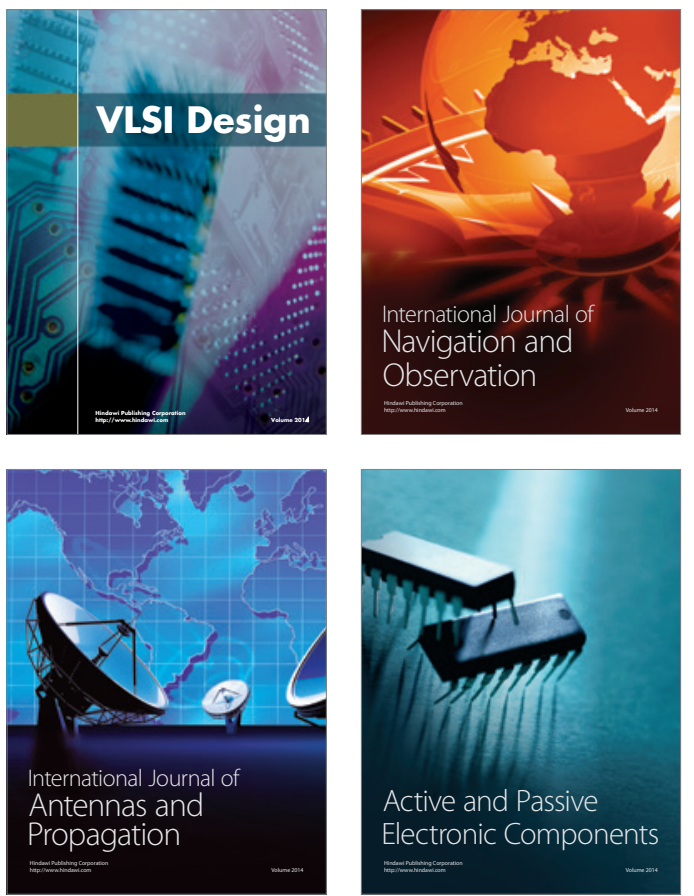
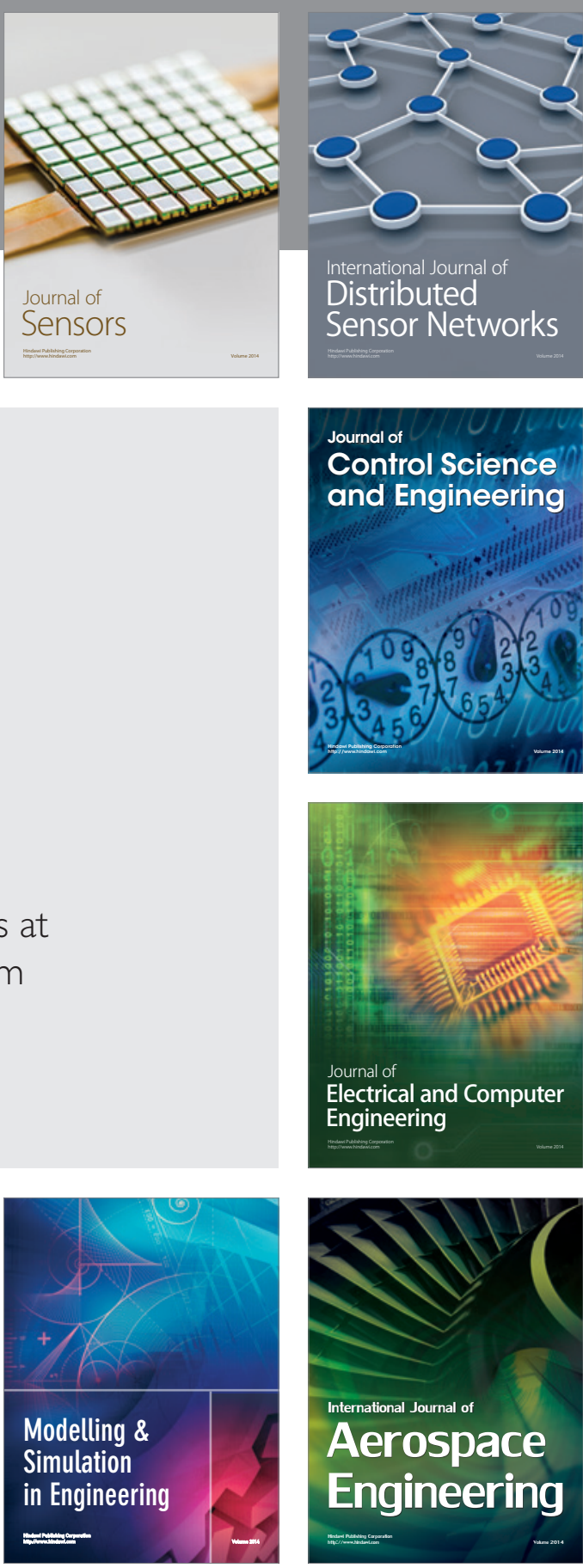

International Journal of

Distributed

Sensor Networks

Journal of

Control Science

and Engineering
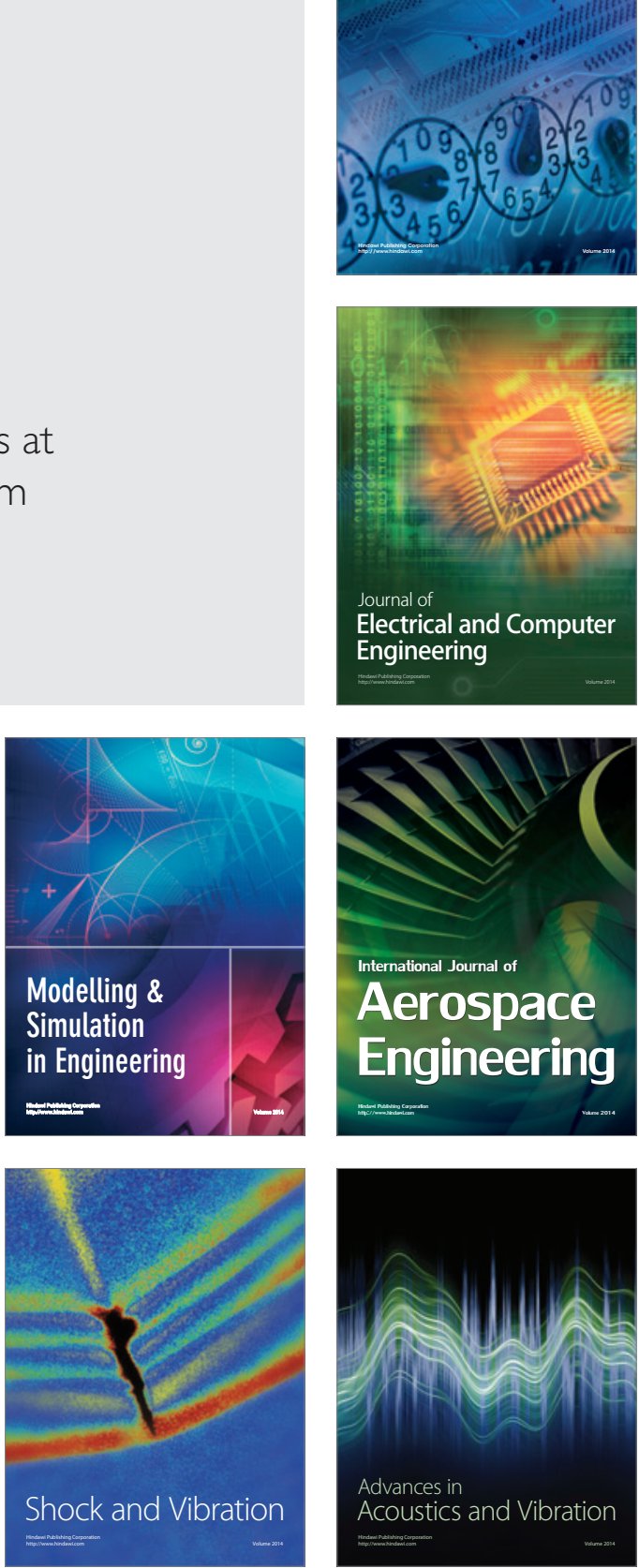